\title{
GGE Biplot Analysis in Thermo Sensitive Genic Male Sterile Lines of Rice (Oryza sativa L.) across Multiple Environments
}

\author{
M. Vinodhini ${ }^{1 *}$, R. Saraswathi ${ }^{1}$, P. L. Viswanathan ${ }^{2}$, S. Arumugachamy ${ }^{3}$, \\ D. Sassikumar ${ }^{4}$ and R. Suresh ${ }^{4}$
}

${ }^{1}$ Department of Rice, ${ }^{2}$ Department of Oilseeds, Tamil Nadu Agricultural University, Coimbatore, Tamil Nadu, India

${ }^{3}$ Rice Research Station, Ambasamudram, Tirunelveli, Tamil Nadu, India

${ }^{4}$ Tamil Nadu Rice Research Institute, Aduthurai, Thanjavur district, Tamil Nadu, India

*Corresponding author

\section{A B S T R A C T}

\section{Keywords}

TGMS, GGE biplot, Pollen sterility \% and spikelet sterility $\%$

\section{Article Info}

Accepted:

17 September 2019

Available Online:

10 October 2019
Stable performance of Thermo-sensitive Genic Male Sterile lines (TGMS) for pollen and spikelet sterility is critical for hybrid seed production in two -line hybrids of rice. The objective of this study is to investigate nine traits in twenty five TGMS lines across three locations viz., Coimbatore (E1), Aduthurai (E2) and Ambasamudram (E3). GGE biplot method was used to analyse the multi-environment data. There was a significant interaction between genotypes and environments for focused traits viz., pollen and spikelet sterility. Among the two weather parameters studied, temperature was found to be the major determinant for sterility induction besides genotypic background. The TGMS lines viz., TNAU 14S, TNAU 100S, TNAU 101S, TNAU $116 \mathrm{~S}$, TNAU 124S, TNAU 135S, TNAU 18S, TNAU 139S, TNAU 143S, TNAU $147 \mathrm{~S}$, TNAU 151S, TNAU 45S, TNAU 46S and TNAU 67S were best performing and stable genotypes in terms of pollen and spikelet sterility\%. Apart from these traits, TNAU 151S and TNAU 124S were found to be stable and best performing for productive tillers, TNAU 139S for panicle exsertion $\%$, TNAU 45S for angle of glume opening, TNAU 67S for stigma exsertion $\%$ and TNAU 46S for stigma exsertion $\%$ and panicle length. In the present study, Aduthurai has been identified as a suitable location for production of two line hybrids apart from Coimbatore.

\section{Introduction}

Rice is considered as the staple food in many parts of the world, including developing countries like Asia, Africa and Latin America. Exploitation of heterosis in rice has significantly contributed to meet out the increasing demand for food (Jiang et al., 
2002). Cytoplasmic male sterile (CMS) system of hybrid seed production is the most widely used system yet it has a major drawback where only $20-30 \%$ of rice germplasm can be used as effective restorers among rice varieties (Singh et al., 2011). On the other hand, the thermosensitive genic male sterility system poses no limitation on the genotypes to be used as male parent and can be exploited in tropical countries like India for production of hybrid rice. However to exploit the TGMS system for hybrid seed production, information on suitable location, time of sowing, genotypes and their flowering time and the prevailing temperatures during the critical phase etc. is required so that fertility reversion of the TGMS line does not occur.

In Tamil Nadu, so far the fertile and sterile phases are being exploited only at two locations viz., Gudalur and Coimbatore respectively. It is important to identify new sites for hybrid seed production in commercial ventures; also genotypic differences exist for stable expression of sterility in TGMS lines. Thus, the objective of the present study is to identify suitable locations for two line hybrid seed production and to identify stable genotypes across multiple environments.

\section{Materials and Methods}

Experiments were conducted in three different locations namely Paddy Breeding StationCoimbatore (E1), Tamil Nadu Rice Research Institute-Aduthurai (E2) and Rice Research Station-Ambasamudram (E3) during Summer 2018. The details of the location are furnished in Table 1. Twenty five TGMS lines viz., TNAU 14S, TNAU 18S, TNAU 45S, TNAU 46S, TNAU 67S, TNAU 71S TNAU 83S, TNAU 84S, TNAU 86S, TNAU 93S, TNAU 100S, TNAU 101S, TNAU 114S, TNAU 115S, TNAU 116S, TNAU 124S, TNAU 135S, TNAU 136S, TNAU 137S, TNAU 139S, TNAU 142S, TNAU 143S, TNAU
145S, TNAU 147S and TNAU 151S from Paddy Breeding Station, Coimbatore constituted the material of study. The lines were sown on $2^{\text {nd }}$ January, 2018 at all the locations and thirty day old seedlings were transplanted in a spacing of $20 \times 20 \mathrm{~cm}$ in Randomized Block Design (RBD) with two replications. Data was recorded on nine characters viz., days to $50 \%$ flowering, plant height $(\mathrm{cm})$, number of productive tillers per plant, panicle length $(\mathrm{cm})$, panicle exsertion $\%$, angle of glume opening $\left({ }^{\circ}\right)$, stigma exsertion $\%$, pollen sterility $\%$ and spikelet sterility \%. (SES, IRRI, 2001).

Data was subjected to GxE biplot analysis for detection of GE interaction using Plant Breeding Tools software version 1.3 (PB Tools, 2013).

\section{Results and Discussion}

The genotype and environment always go hand in hand for a plant breeder to reduce the disparity in selection of superior/ideal genotypes during cultivar development. As most of the traits have quantitative inheritance, understanding their Genotype $\mathrm{x}$ Environment Interaction (GEI) will be helpful in making decisions for deployment of genotype(s) in specific/wider environments (Ebdon and Gauch, 2002, Kang et al., 2004). Thus GE is both an opportunity and challenge for the plant breeders.

There are many standard univariate and multivariate methods to evaluate the $\mathrm{G} \times \mathrm{E}$ viz., joint linear regression, singular value decomposition, Additive Main effects Multiplicative Interaction (AMMI) (Kang, 2002). The gap between quantification of GE and matching genotypes with environments was partially bridged by biplot analysis methodology of Gabriel (1971). Later Yan et al., (2000) devised a comprehensive graphical method in which genotype and genotype 
environment interaction are simultaneously considered for appropriate genotype in test environment evaluation, popularly termed as 'GGE biplot' method. This visual method is simple but informative even for researchers with limited training in statistics and computer applications (Yan and Tinker, 2006).

GGE biplot shows the first two principal components (PC1 and PC2) which are obtained by decomposition of singular values of multi-location trials data for a particular trait. It enables the identification of stable genotypes in different environments and also comparison of their yield performances in different environments, identification of socalled 'ideal' genotype, as well as 'megaenvironments' (Mitrovic et al., 2012). It is also a useful tool for identifying locations with optimized cultivar performance and for making better use of limited resources available for the testing program (Khalil et al., 2011).

In our study, the foremost criterion for a TGMS line to be exploited for hybrid seed production is its stability in expression of pollen and spikelet sterility during the critical stage. Thus, the variance analysis for the two focused traits (Table 2) revealed significant interaction between genotypes and environments and thus, the need to identify suitable genotypes $v s$. locations.

\section{Mean performance and stability of genotypes}

The mean data across three locations for nine traits is given in Table $3 \mathrm{a}$ and Table $3 \mathrm{~b}$. The flowering period for the TGMS lines was from $1^{\text {st }}$ April to $26^{\text {th }}$ April at Coimbatore, $14^{\text {th }}$ March to $24^{\text {th }}$ April at Aduthurai and $11^{\text {th }}$ March to $5^{\text {th }}$ April at Ambasamudram respectively. The maximum, minimum temperature and relative humidity recorded during the flowering was $30.9^{\circ} \mathrm{C}, 20.4^{\circ} \mathrm{C}$ and
$87.6 \%$ at Coimbatore, $34.1^{\circ} \mathrm{C}, 22.9^{\circ} \mathrm{C}$ and $95 \%$ at Aduthurai and $34.5^{\circ} \mathrm{C}, 23.5^{\circ} \mathrm{C}$ and $25.9 \%$ at Ambasamudram. It has been pointed out by Liu et al., (1997) that fertility varies with different genetic background and the primary factor which alters the fertility in TGMS lines is the temperature whereas relative humidity is one of the secondary factors. At Coimbatore, the maximum and minimum temperatures were lower than that at other locations. Hence six genotypes viz., G5, G6, G14, G16, G24 and G25 showed fertile pollen grains but were $100 \%$ sterile at other two locations. The lines G2 and G23 are to be rejected because they did not conform to TGMS line behavior. One genotype G10 expressed only 41.5 per cent pollen sterility at Ambasamudram while it was 100 per cent sterile at other locations. Reduction in sterility may be due to decrease in RH (25.9\% only as against $87.6 \%$ and $95 \%$ at other two locations) as it causes a reduction in the spikelet temperature than the corresponding atmospheric/ air temperature (Weerakoon et al., 2008).

The ranking of 25 genotypes based on their mean and stability of performance and relative to an ideal genotype across three environments is presented in Figure 1. Genotypes with high mean performance (except flowering and plant height) and stability are the desirable ones in stability analysis. Genotypes present in the concentric area were stable genotypes compared to those laid outside (Yan and Hunt, 2002). Accordingly, in our study, the following genotypes (Table 4) were identified as stable for each trait.

Khodadad et al., (2011) identified three genotypes viz., G12, G14 and G4 as best based on mean and stability out of the 14 genotypes tested in nine environments. Nassir and Ariyo (2011) identified TOX 3107 as having a combination of stable and average yield when grown in tropical inland swamp. Using 
Average Environment Tester coordinate, Akter et al., (2015) identified three rice hybrids viz., BRRI10A/BRRI10R (G2), IR58025A/BRRI10R (G3) and BRRI hybrid dhan1 (G4) to be stable over five environments with high mean. Susanto et al., (2015) reported that G3 (A69-1) was highly stable with high grain yield in all the five environments tested based on mean performance and stability. Two genotypes G3(14S) and G6(166S) were reported as stable across three seasons with high mean performance for yield traits in backcross introgression lines of Oryza sativa cv.Swarna/Oryza nivara by Divya Balakrishnan et al., (2016)

\section{GGE biplot polygon view for ideal genotype}

The polygon view of a biplot is one of the best way to visualize the interaction patterns between genotypes and environments and to interpret a biplot effectively (Yan and Kang, 2003). In this, some of the genotypes are placed on the crests, while the rest are surrounded by the polygon. Those at the crests of the polygon represent the vertex/ideal genotype. As the genotypes placed on the peak had the longest detachment from the biplot origin they were expected to be the most responsive.

The Table 5 represents the ideal genotypes for each environment for each trait extracted from the biplot polygon view. For pollen sterility $\%$, the environment E1 (Coimbatore), E2 (Aduthurai) and E3 (Ambasamudram) fell in three different sectors (Figure 2). The two principal components (PC1 and PC2) accounted for $86.9 \%$ of the total variation, of which PC1 explained $66.4 \%$ and PC2 explained 20.5\%. Genotypes G10, G11 in E1 (Coimbatore), G1, G3, G4, G6, G7, G8, G9, G12, G13, G14, G15, G17, G18, G19, G20 and G21 in E2 (Aduthurai) and G5 in E3 (Ambasamudram) were the vertex genotype and are appropriate for growing in the respective environments.

In case of spikelet sterility \%, Environment E1 (Coimbatore), E2 (Aduthurai) and E3 (Ambasamudram) fell in three different sectors (Figure 2). The two principal components (PC1 and PC2) accounted for $96.4 \%$ of the total variation, of which $\mathrm{PC} 1$ explained $74.3 \%$ and PC2 explained $22.1 \%$ respectively. The genotype $\mathrm{G} 11$ in $\mathrm{E} 1$ (Coimbatore), genotypes G1, G3, G4, G6, G7, G8, G9, G12, G13, G14, G15, G17, G18, G19, G20, G21, G22 and G24 in E2 (Aduthurai) and genotype G5 in E3 (Ambasamudram) was the vertex genotype and are appropriate for growing in the respective environments.

Sairekha et al., (2018) identified TNAU 45S (G6), TNUAU 60S (G1) and TNAU 95S (G8) as winning genotypes for pollen and spikelet sterility at Aduthurai (E1), GDR 70S (G2), TNAU 14S (G3) and TNAU 18S (G4) at Ambasamudram (E2) and TNAU 39S (G5) at Coimbatore (E3) environment among eight TGMS lines studied.

One of the important floral traits which effect the outcrossing percentage is male sterility. Apart from male sterility, the outcrossing percentage in female is influenced by several other floral traits which include stigma exsertion and angle of glume opening (Virmani, 1994).

In case of angle of glume opening, environments fell in two different sectors with the genotypes G15 and G19 at the vertex in environment E1 (Coimbatore) and E2 (Aduthurai) while G18 was at the vertex in environment E3 (Ambasamudram). For stigma exsertion, G7 and G20 in environment E1 (Coimbatore), E3 (Ambasamudram) and G10 in environment E2 (Aduthurai) were the vertex genotypes and are appropriate for growing in the respective environments. 
Table.1 Agroclimatic zone, Latitude, longitude and weather data of the three locations

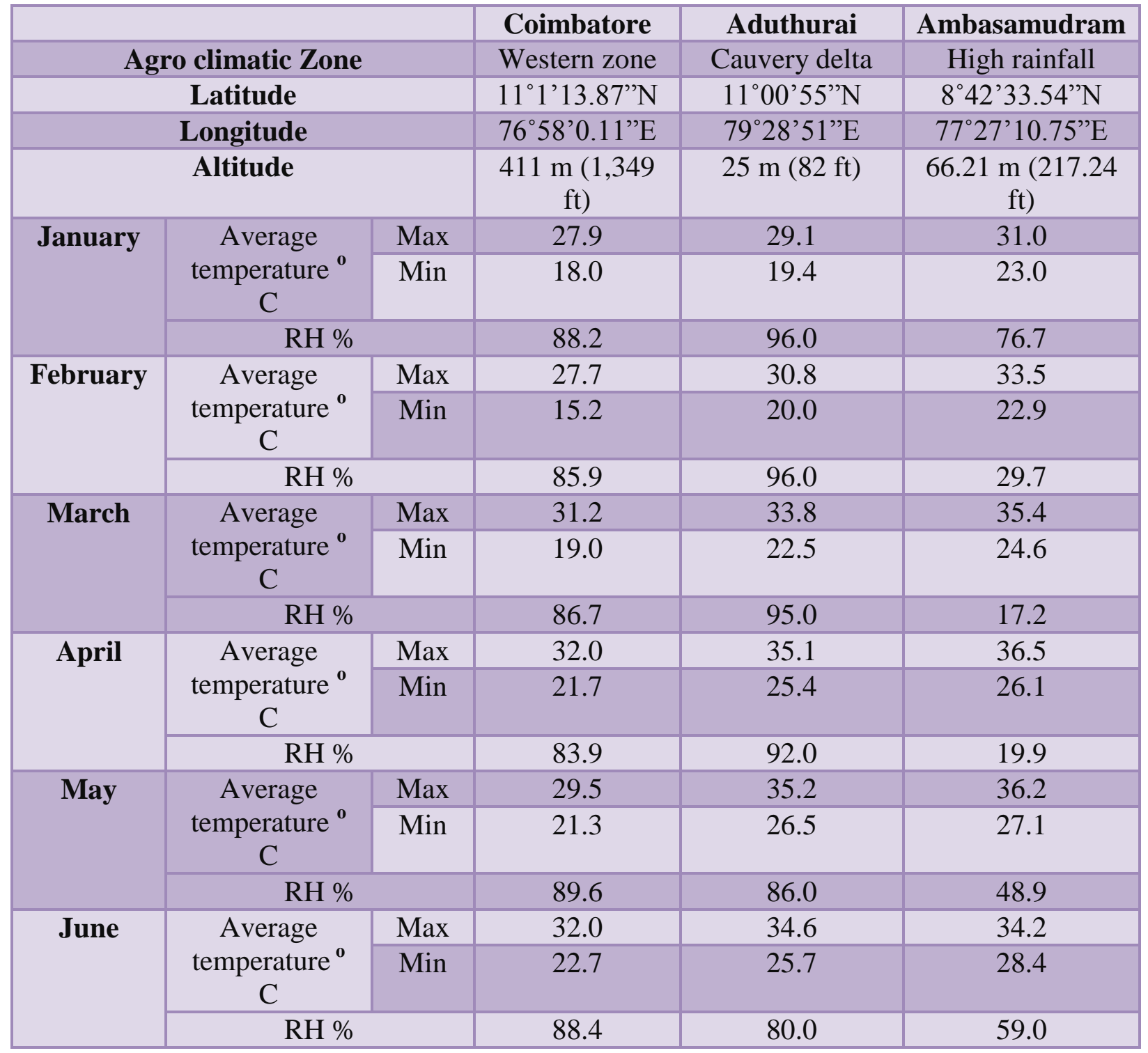

Table.2 Combined analysis of variance over three environments for pollen and spikelet sterility in 25 TGMS lines of rice

\begin{tabular}{|c|c|c|c|}
\hline Source & Degree of & \multicolumn{2}{|c|}{ Mean Square (MS) } \\
\cline { 3 - 4 } & $\begin{array}{c}\text { freedom } \\
\text { (Df) }\end{array}$ & $\begin{array}{c}\text { Pollen } \\
\text { sterility }\end{array}$ & $\begin{array}{c}\text { Spikelet } \\
\text { sterility }\end{array}$ \\
\hline Genotype & 24 & $1137.89 * *$ & $1992.60 * *$ \\
\hline Environment & 2 & $943.29 * *$ & $261.89 * *$ \\
\hline Genotype $\times$ Environment & 48 & $543.96 * *$ & $632.03 * *$ \\
\hline Error & 75 & 4.94 & 5.04 \\
\hline Total & 149 & & \\
\hline
\end{tabular}

** Significant at $1 \%$ level of probability 
Table.3a Mean performance of twenty TGMS lines in rice across three locations for days to 50\% flowering, plant height, productive tillers per plant, panicle length and panicle exsertion

\begin{tabular}{|c|c|c|c|c|c|c|c|c|c|c|c|c|c|c|c|c|}
\hline \multirow[t]{2}{*}{$\begin{array}{l}\text { Genotype } \\
\text { code }\end{array}$} & \multirow[t]{2}{*}{ Genotype } & \multicolumn{3}{|c|}{$\begin{array}{c}\text { Days to } 50 \% \\
\text { flowering }\end{array}$} & \multicolumn{3}{|c|}{ Plant height $(\mathrm{cm})$} & \multicolumn{3}{|c|}{$\begin{array}{c}\text { Productive tillers } \\
\text { per plant }\end{array}$} & \multicolumn{3}{|c|}{$\begin{array}{c}\text { Panicle length } \\
\text { (cm) }\end{array}$} & \multicolumn{3}{|c|}{$\begin{array}{c}\text { Panicle } \\
\text { exsertion \% }\end{array}$} \\
\hline & & E1 & E2 & E3 & E1 & E2 & E3 & E1 & E2 & E3 & E1 & E2 & E3 & E1 & E2 & E3 \\
\hline G1 & TNAU 14S & 112.0 & 107.0 & 94.5 & 87.4 & 78.5 & 63.2 & 21.8 & 16.3 & 14.1 & 18.7 & 18.8 & 17.2 & 88.7 & 80.1 & 74.2 \\
\hline G2 & TNAU 93S & 116.0 & 109.0 & 93.5 & 84.2 & 80.0 & 79.9 & 15.7 & 8.6 & 18.7 & 21.2 & 25.5 & 22.5 & 84.0 & 82.2 & 83.4 \\
\hline G3 & TNAU 100S & 112.0 & 98.0 & 96.5 & 82.7 & 75.5 & 72.2 & 27.7 & 12.8 & 19.0 & 20.8 & 21.4 & 20.8 & 77.6 & 72.5 & 81.2 \\
\hline G4 & TNAU 101S & 111.5 & 88.5 & 89.5 & 79.9 & 73.5 & 81.5 & 22.5 & 11.9 & 21.1 & 16.4 & 28.1 & 25.4 & 80.4 & 74.5 & 84.1 \\
\hline & TNAU 114S & 107.0 & 110.5 & 87.0 & 80.6 & 71.4 & 71.8 & 30.9 & 11.0 & 22.4 & 22.8 & 20.9 & 22.9 & 85.8 & 76.0 & 78.8 \\
\hline G6 & TNAU $115 \mathrm{~S}$ & 103.5 & 111.5 & 89.5 & 76.1 & 62.6 & 85.3 & 29.7 & 10.9 & 24.0 & 16.5 & 22.6 & 23.5 & 80.8 & 78.5 & 83.4 \\
\hline G7 & TNAU $116 \mathrm{~S}$ & 105.0 & 112.5 & 88.5 & 75.5 & 69.2 & 86.8 & 29.7 & 9.1 & 26.7 & 19.7 & 21.1 & 23.3 & 77.6 & 78.0 & 80.1 \\
\hline & & & & 91.5 & & & & & & & 17.4 & 18.0 & 22.4 & 79.0 & 69.9 & 78.7 \\
\hline G9 & TNAU 135S & 117.5 & 103.5 & 89.5 & 92.4 & 82.6 & 82.1 & 22.7 & 11.1 & 21.3 & 21.2 & 22.3 & 23.5 & 82.4 & 74.0 & 74.2 \\
\hline G10 & & & & & & & & & & & 24.3 & 26.6 & 22.1 & 82.2 & 73.0 & 81.7 \\
\hline G11 & TNAU $137 \mathrm{~S}$ & 103.0 & 79.0 & 79.0 & 92.1 & 80.7 & 72.4 & 30.6 & 12.3 & 18.4 & 21.2 & 24.4 & 25.0 & 83.1 & 76.9 & 78.5 \\
\hline & & & & & & & & & & & 21.0 & 25.0 & 21.8 & 84.0 & 72.5 & 78.1 \\
\hline G13 & J 139S & 95.0 & 110.0 & 74.0 & 68.0 & 70.0 & 81.9 & 23.5 & 10.6 & 21.2 & 19.2 & 21.4 & 20.5 & 83.8 & 76.8 & 81.4 \\
\hline G14 & TNAU $142 \mathrm{~S}$ & 104.0 & 87.0 & 89.0 & 92.9 & 70.6 & 87.8 & 25.7 & 16.1 & 25.6 & 21.0 & 19.9 & 25.0 & 84.4 & 76.1 & 82.6 \\
\hline G15 & TNAU $143 \mathrm{~S}$ & 103.0 & 88.0 & 87.5 & 87.3 & 73.7 & 86.1 & 25.2 & 21.5 & 17.5 & 21.5 & 22.5 & 21.0 & 83.0 & 80.3 & 74.4 \\
\hline G16 & TNAU $145 \mathrm{~S}$ & 104.0 & 104.0 & 98.0 & 91.4 & 75.7 & 90.5 & 24.1 & 16.0 & 20.8 & 25.5 & 21.8 & 22.0 & 81.7 & 82.9 & 80.7 \\
\hline G17 & TNAU $147 \mathrm{~S}$ & 117.0 & 110.5 & 95.5 & 83.0 & 77.9 & 64.1 & 26.2 & 18.4 & 15.9 & 20.0 & 19.1 & 17.5 & 82.5 & 77.7 & 78.1 \\
\hline G18 & TNAU $151 \mathrm{~S}$ & 111.0 & 115.0 & 96.5 & 91.1 & 82.0 & 79.2 & 28.0 & 13.5 & 20.7 & 18.6 & 22.1 & 19.9 & 79.1 & 76.3 & 78.0 \\
\hline G19 & TNAU 45S & & 107.0 & 97.0 & 58.9 & 75.1 & 65.8 & 24.9 & 15.1 & 17.5 & 20.9 & 23.8 & 21.5 & 74.9 & 73.5 & 79.3 \\
\hline G20 & TNAU $46 \mathrm{~S}$ & 112.0 & 101.0 & 89.5 & 77.5 & 74.4 & 68.3 & 26.7 & 14.2 & 21.8 & 24.5 & 27.3 & 22.6 & 83.5 & 76.5 & 83.5 \\
\hline G21 & & & & 95.5 & 77.3 & 75.7 & & 22.1 & 13.9 & 21.4 & 26.3 & 25.7 & 21.9 & 81.7 & 78.7 & 68.8 \\
\hline G22 & TNAU 71S & 121.5 & 108.0 & 96.0 & 83.7 & 85.3 & 75.9 & 27.8 & 12.3 & 17.1 & 27.8 & 27.0 & 28.2 & 80.0 & 73.3 & 78.9 \\
\hline G23 & & & & 93.5 & 93.7 & 76.1 & 69.1 & 26.1 & 14.05 & 24.1 & 20.6 & 21.9 & 18.0 & 74.1 & 73.9 & 77.0 \\
\hline G24 & TNAU 84S & 104.5 & 107.5 & 90.5 & 85.6 & 78.8 & 72.9 & 28.0 & 12.8 & 17.5 & 15.7 & 20.3 & 17.9 & 88.4 & 77.6 & 81.5 \\
\hline G25 & TNAU 86S & 112.5 & 110.0 & 98.0 & 90.9 & 73.5 & 77.2 & 31.5 & 10.2 & 18.2 & 19.5 & 22.2 & 19.5 & 78.9 & 76.5 & 82.1 \\
\hline
\end{tabular}


Table.3b Mean performance of twenty five TGMS lines in rice across three locations for angle of glume opening, stigma exsertion, pollen sterility and spikelet sterility

\begin{tabular}{|c|c|c|c|c|c|c|c|c|c|c|c|c|c|}
\hline \multirow[t]{2}{*}{$\begin{array}{l}\text { Genotype } \\
\text { code }\end{array}$} & \multirow[t]{2}{*}{ Genotype } & \multicolumn{3}{|c|}{$\begin{array}{c}\text { Angle of glume } \\
\text { opening }\left({ }^{0}\right)\end{array}$} & \multicolumn{3}{|c|}{$\begin{array}{c}\text { Stigma exsertion } \\
\%\end{array}$} & \multicolumn{3}{|c|}{ Pollen sterility \% } & \multicolumn{3}{|c|}{ Spikelet sterility\% } \\
\hline & & E1 & E2 & E3 & E1 & E2 & E3 & E1 & E2 & $\mathbf{E 3}$ & E1 & E2 & E3 \\
\hline G1 & TNAU $14 \mathrm{~S}$ & 22.5 & 22.5 & 28.5 & 51.6 & 47.0 & 46.6 & 100.0 & 100.0 & 100.0 & 100.0 & 100.0 & 100.0 \\
\hline G2 & TNAU 93S & 24.0 & 23.0 & 24.5 & 31.9 & 18.9 & 29.8 & 28.2 & 26.2 & 16.5 & 74.8 & 40.1 & 30.2 \\
\hline G3 & TNAU 100S & 20.5 & 28.0 & 25.0 & 47.4 & 34.5 & 39.9 & 100.0 & 100.0 & 100.0 & 100.0 & 100.0 & 100.0 \\
\hline G4 & TNAU 101S & 22.0 & 28.5 & 23.0 & 48.5 & 35.6 & 40.2 & 100.0 & 100.0 & 100.0 & 100.0 & 100.0 & 100.0 \\
\hline G5 & TNAU 114S & 27.0 & 26.5 & 22.5 & 30.3 & 31.0 & 34.8 & 39.5 & 100.0 & 100.0 & 70.8 & 50.3 & 100.0 \\
\hline G6 & TNAU $115 \mathrm{~S}$ & 25.5 & 23.5 & 25.0 & 50.5 & 40.1 & 41.9 & 99.1 & 100.0 & 100.0 & 99.1 & 100.0 & 100.0 \\
\hline G7 & TNAU 116S & 26.5 & 32.0 & 22.5 & 66.1 & 50.2 & 49.3 & 100.0 & 100.0 & 100.0 & 100.0 & 100.0 & 100.0 \\
\hline G8 & TNAU 124S & 25.0 & 20.0 & 24.0 & 54.2 & 60.9 & 47.0 & 100.0 & 100.0 & 100.0 & 100.0 & 100.0 & 100.0 \\
\hline G9 & TNAU 135S & 18.5 & 15.0 & 18.0 & 34.0 & 25.2 & 36.2 & 100.0 & 100.0 & 100.0 & 100.0 & 100.0 & 100.0 \\
\hline G10 & TNAU 136S & 21.0 & 27.5 & 24.5 & 47.1 & 58.6 & 55.3 & 100.0 & 100.0 & 41.5 & 100.0 & 100.0 & 88.0 \\
\hline G11 & TNAU 137S & 26.5 & 24.5 & 22.0 & 60.2 & 45.0 & 43.6 & 100.0 & 55.0 & 12.8 & 100.0 & 100.0 & 100.0 \\
\hline G12 & TNAU 18S & 25.0 & 23.5 & 24.0 & 45.9 & 42.7 & 37.2 & 100.0 & 100.0 & 100.0 & 100.0 & 100.0 & 100.0 \\
\hline G13 & TNAU 139S & 21.5 & 27.5 & 25.5 & 23.1 & 33.1 & 23.7 & 100.0 & 100.0 & 100.0 & 100.0 & 100.0 & 100.0 \\
\hline G14 & TNAU $142 \mathrm{~S}$ & 25.5 & 21.0 & 22.5 & 45.4 & 42.3 & 37.5 & 99.7 & 100.0 & 100.0 & 100.0 & 100.0 & 100.0 \\
\hline G15 & TNAU 143S & 27.0 & 32.5 & 24.0 & 42.4 & 35.4 & 27.6 & 100.0 & 100.0 & 100.0 & 100.0 & 100.0 & 100.0 \\
\hline G16 & TNAU $145 \mathrm{~S}$ & 20.5 & 21.5 & 22.0 & 52.1 & 51.2 & 45.4 & 92.7 & 100.0 & 100.0 & 89.5 & 100.0 & 100.0 \\
\hline G17 & TNAU 147S & 20.5 & 20.0 & 26.5 & 46.6 & 38.9 & 49.3 & 100.0 & 100.0 & 100.0 & 100.0 & 100.0 & 100.0 \\
\hline G18 & TNAU 151S & 23.0 & 15.5 & 28.0 & 47.7 & 42.8 & 54.2 & 100.0 & 100.0 & 100.0 & 100.0 & 100.0 & 100.0 \\
\hline G19 & TNAU 45S & 28.5 & 27.5 & 24.5 & 54.8 & 43.4 & 46.0 & 100.0 & 100.0 & 100.0 & 100.0 & 100.0 & 100.0 \\
\hline G20 & TNAU 46S & 26.0 & 23.5 & 21.5 & 61.4 & 54.6 & 59.7 & 100.0 & 100.0 & 100.0 & 100.0 & 100.0 & 100.0 \\
\hline G21 & TNAU 67S & 18.0 & 18.5 & 22.0 & 54.8 & 45.9 & 54.6 & 100.0 & 100.0 & 100.0 & 100.0 & 100.0 & 100.0 \\
\hline G22 & TNAU 71S & 17.0 & 19.5 & 19.5 & 33.2 & 25.5 & 29.3 & 100.0 & 38.0 & 100.0 & 100.0 & 98.0 & 100.0 \\
\hline G23 & TNAU 83S & 22.5 & 21.0 & 25.0 & 27.6 & 32.7 & 27.4 & 80.1 & 100.0 & 35.4 & 100.0 & 100.0 & 94.6 \\
\hline G24 & TNAU 84S & 21.5 & 21.0 & 25.5 & 52.1 & 43.9 & 37.0 & 94.7 & 100.0 & 100.0 & 100.0 & 100.0 & 100.0 \\
\hline G25 & TNAU 86S & 22.0 & 20.0 & 22.5 & 51.0 & 46.6 & 52.9 & 48.8 & 100.0 & 100.0 & 65.1 & 100.0 & 100.0 \\
\hline
\end{tabular}


Table.4 Stable TGMS lines for different traits in rice

\begin{tabular}{|c|c|c|}
\hline Trait & Stable genotypes & $\begin{array}{c}\text { Mean } \\
\text { performance }\end{array}$ \\
\hline \multirow{5}{*}{$\begin{array}{c}\text { Days to } 50 \% \text { flowering } \\
\text { (days) }\end{array}$} & G17 & 107.6 \\
\hline & G12 & 105.8 \\
\hline & G25 & 106.8 \\
\hline & $\mathrm{G} 2$ & 106.2 \\
\hline & $\mathrm{G} 23$ & 105.5 \\
\hline \multirow[t]{3}{*}{ Plant height (cm) } & G9 & 85.7 \\
\hline & G18 & 84.1 \\
\hline & G25 & 80.53 \\
\hline \multirow{3}{*}{ Productive tillers per plant } & G25 & 19.9 \\
\hline & G11 & 20.4 \\
\hline & G8 & 22.3 \\
\hline \multirow[t]{3}{*}{ Panicle length $(\mathrm{cm})$} & G22 & 27.7 \\
\hline & G20 & 24.8 \\
\hline & G10 & 24.3 \\
\hline \multirow[t]{5}{*}{ Panicle exsertion (\%) } & G2 & 83.2 \\
\hline & G24 & 82.5 \\
\hline & G16 & 81.8 \\
\hline & G14 & 81.0 \\
\hline & G13 & 80.7 \\
\hline Angle of glume opening $\left({ }^{\circ}\right)$ & G19 & 26.8 \\
\hline \multirow[t]{4}{*}{ Stigma exsertion $(\%)$} & G20 & 58.6 \\
\hline & G21 & 51.8 \\
\hline & G25 & 50.2 \\
\hline & G16 & 49.6 \\
\hline Pollen sterility (\%) & $\begin{array}{l}\text { G1, G3, G4, G7, G8, G9, } \\
\text { G10, G12, G13, G15, G17, } \\
\text { G18, G19, G20 and G21 }\end{array}$ & 100 \\
\hline Spikelet sterility (\%) & $\begin{array}{c}\mathrm{G} 1, \mathrm{G} 3, \mathrm{G} 4, \mathrm{G} 7, \mathrm{G} 8, \mathrm{G} 9 \\
\mathrm{G} 12, \mathrm{G} 13, \mathrm{G} 14, \mathrm{G} 15, \mathrm{G} 17, \\
\mathrm{G} 18, \mathrm{G} 19, \mathrm{G} 20, \mathrm{G} 21 \text { and } \mathrm{G} 24\end{array}$ & 100 \\
\hline
\end{tabular}


Table.5 Ideal TGMS lines for different traits at different environments

\begin{tabular}{|c|c|c|}
\hline Trait & Environment & Vertex genotypes \\
\hline Days to 50\% flowering & E1 (Coimbatore) and E3 (Ambasamudram) & G19 \\
\cline { 2 - 3 } & E2 (Aduthurai) & G18 \\
\hline Plant height & E1 (Coimbatore) and E3 (Ambasamudram) & G14 and G16 \\
\cline { 2 - 3 } $\begin{array}{c}\text { Productive tillers per } \\
\text { plant }\end{array}$ & E1 (Aduthurai) & G1 \\
\cline { 2 - 3 } Panicle length & E1 (Coimbatore) and E3 (Ambasamudram) & G7 \\
\hline \multirow{2}{*}{ Panicle exsertion } & E3 (Aduthurai) & G15 \\
\hline \multirow{2}{*}{ Angle of glume opening } & E1 (Coimbatore) and E2 (Aduthurai) & G16 and G22 \\
\hline \multirow{2}{*}{ Stigma exsertion } & E3 (Ambasamudram) & G4 \\
\cline { 2 - 3 } & E1 (Coimbatore) and E2 (Aduthurai) & G1 \\
\hline & E3 (Ambasamudram) & G15 and G19 \\
\hline
\end{tabular}

Fig.1 Average Environment Axis (AEA) view of GGE biplot showing the mean performance and stability of genotypes

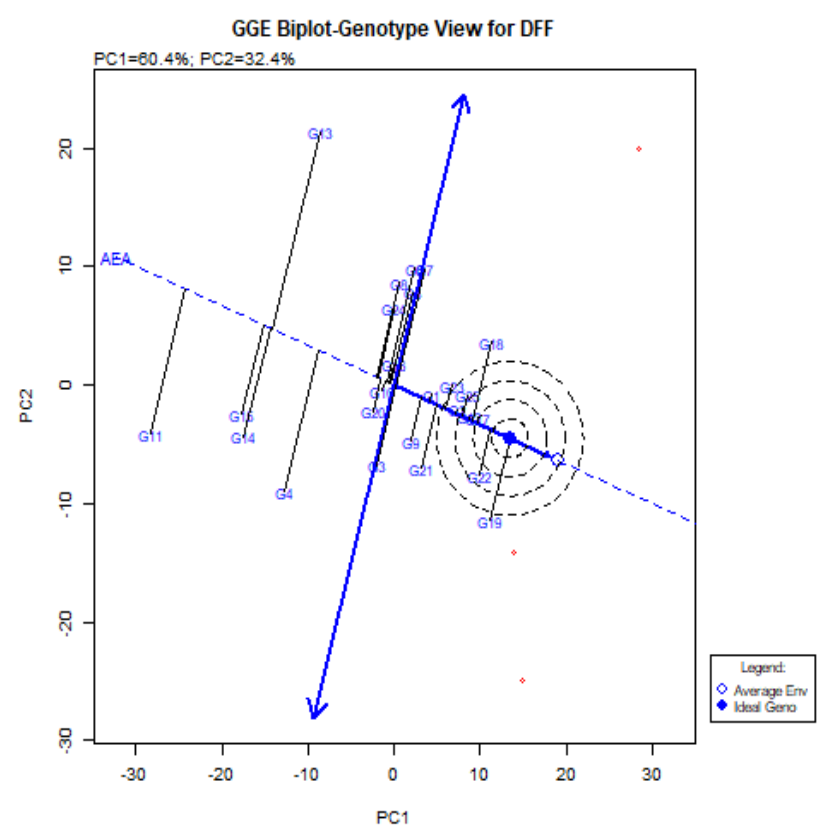

a) Days to $\mathbf{5 0 \%}$ flowering

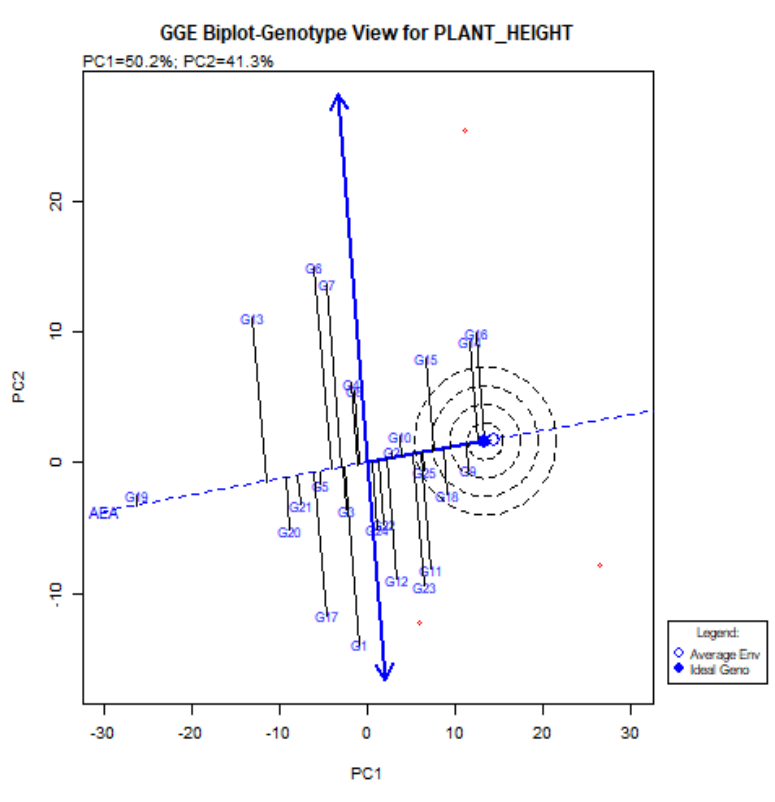

b) Plant height 


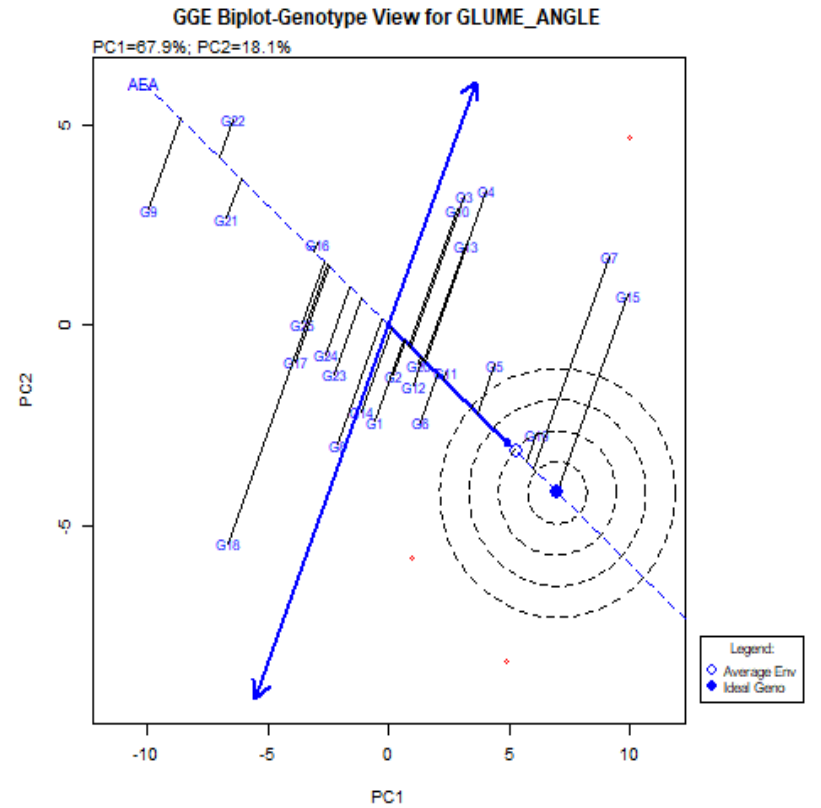

c) Angle of glume opening

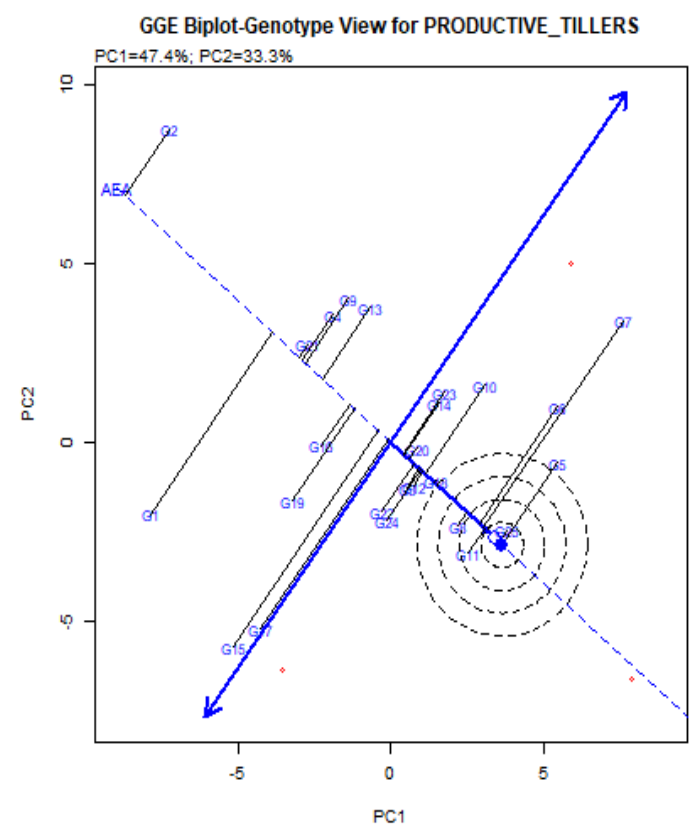

e) Productive tillers

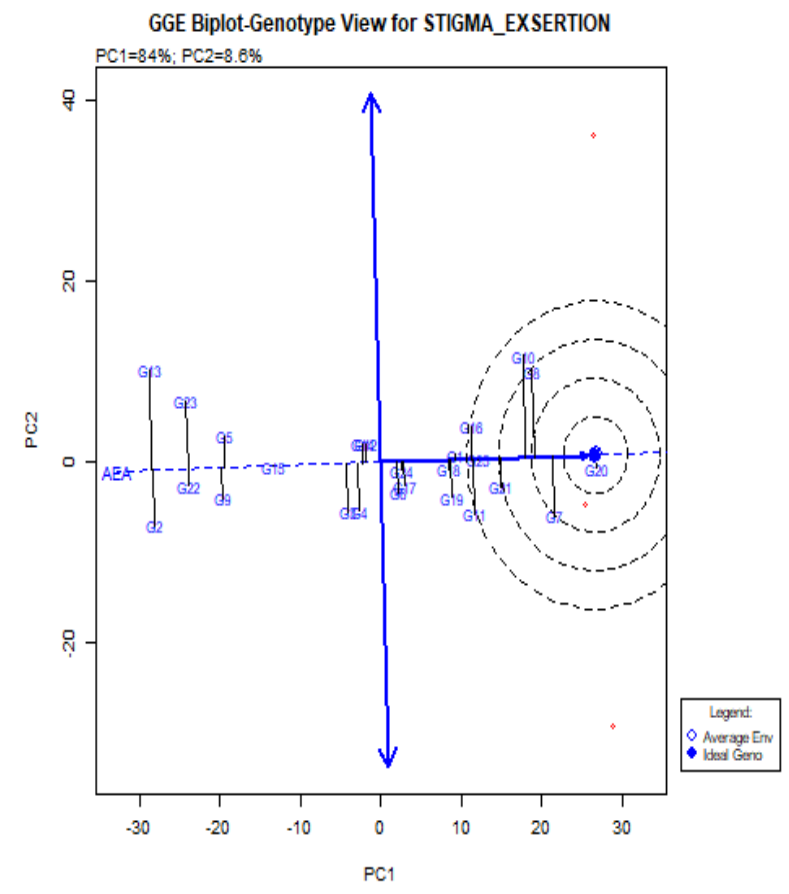

d) Stigma exsertion

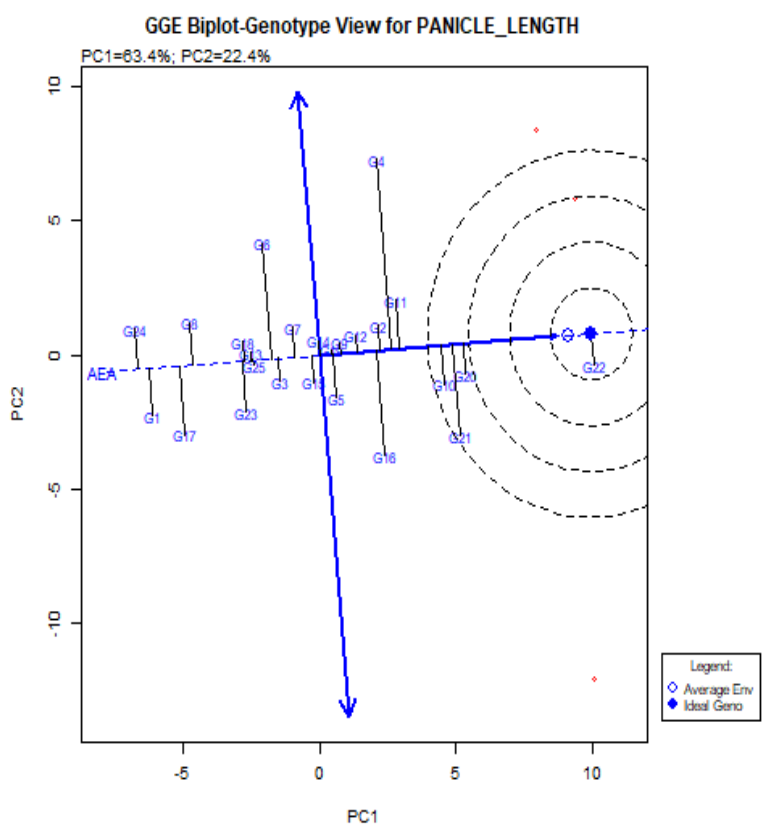

f) Panicle length 


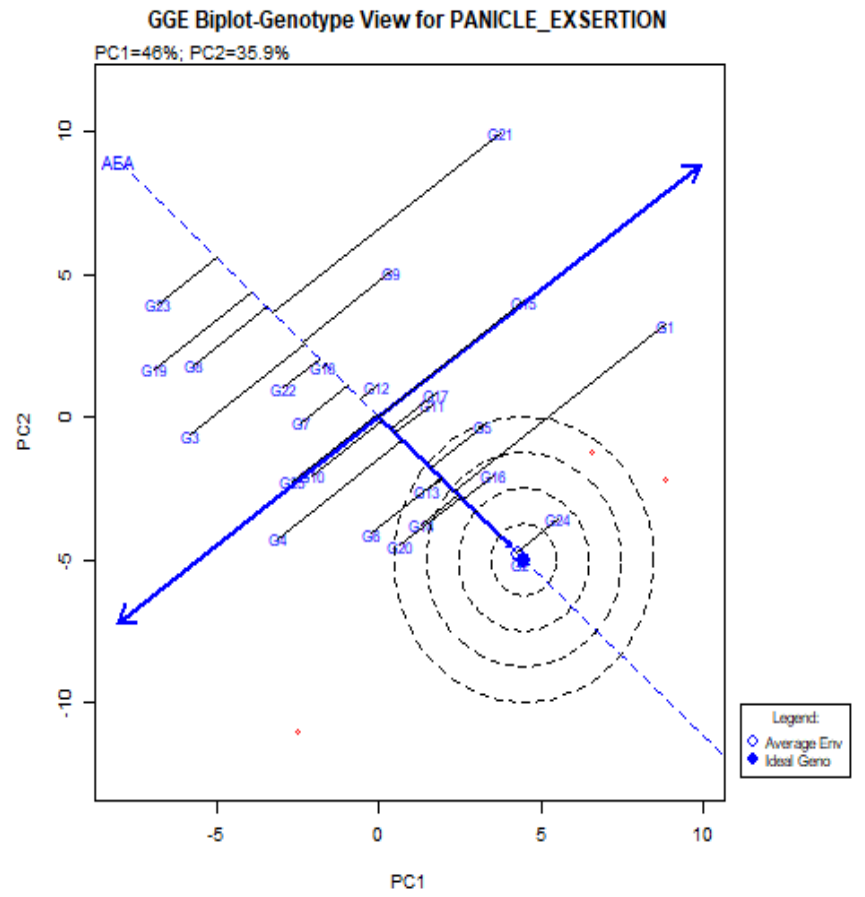

g) Panicle exsertion

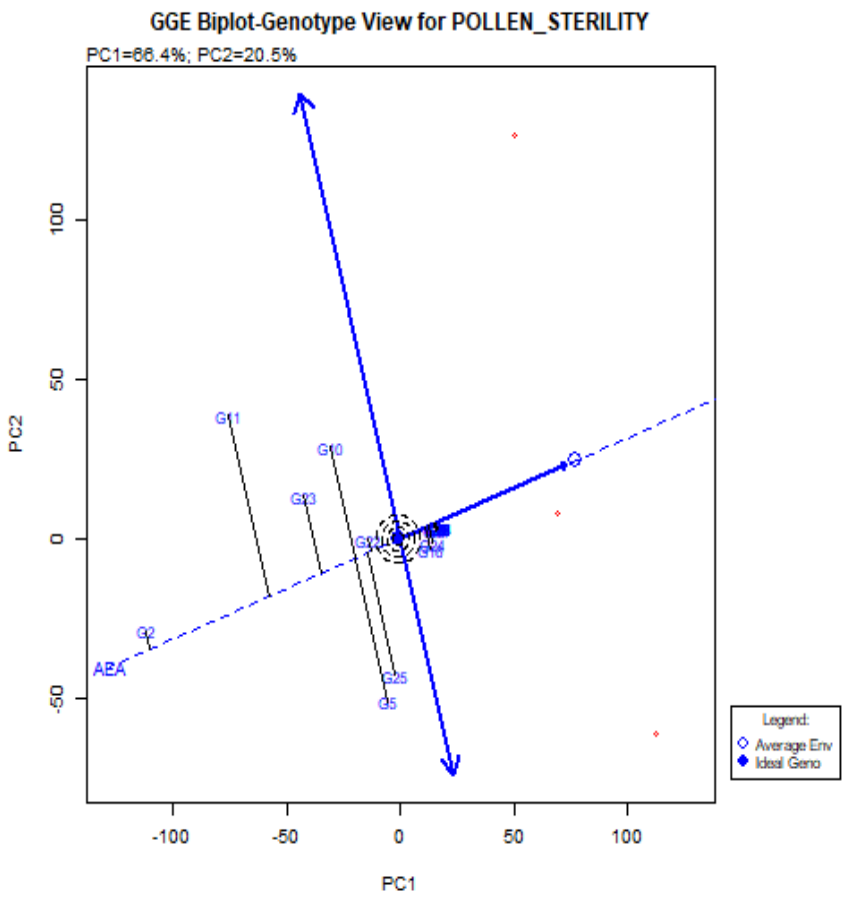

h) Pollen sterility

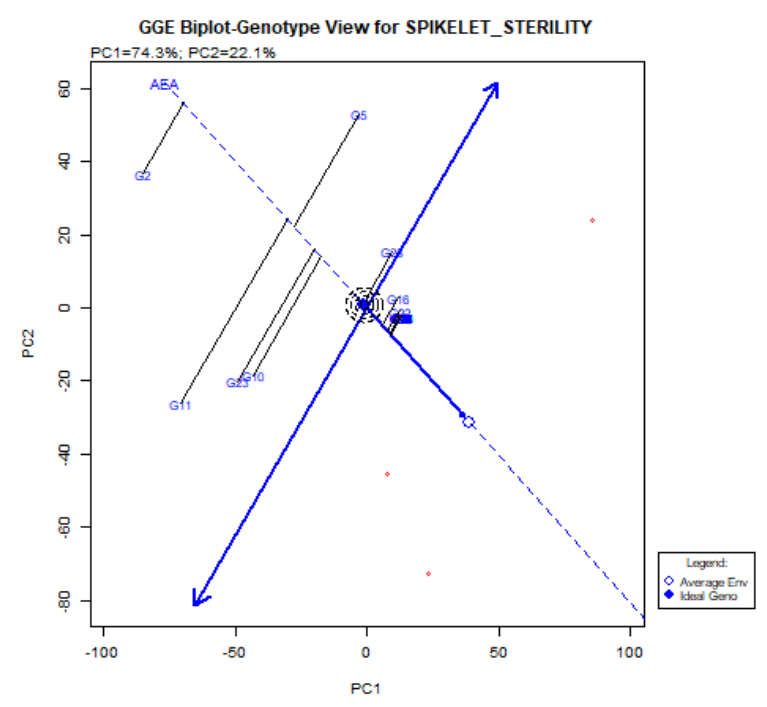

i) Spikelet sterility 
Fig.2 Polygon view of GGE biplot for different traits in TGMS lines

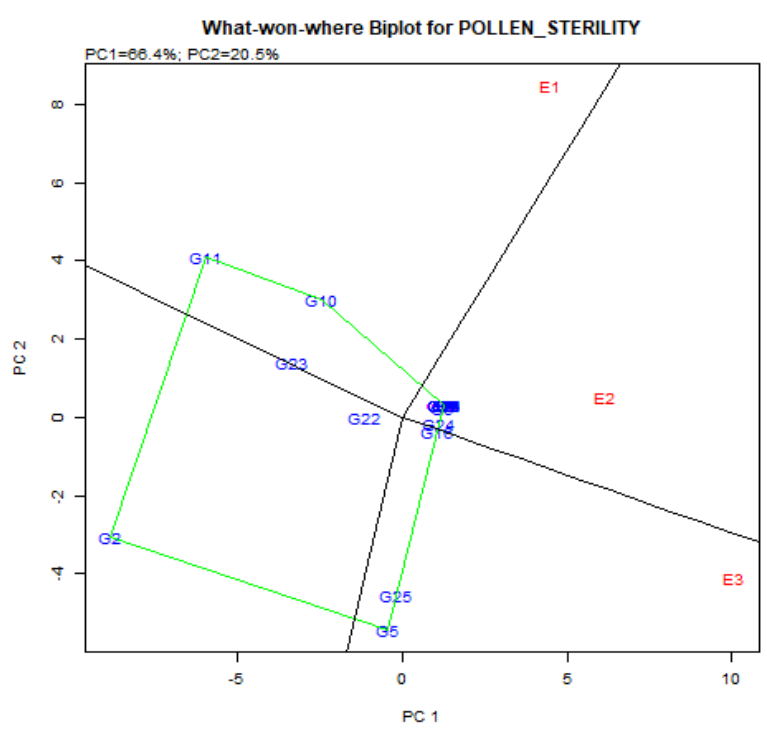

a) Pollen sterility

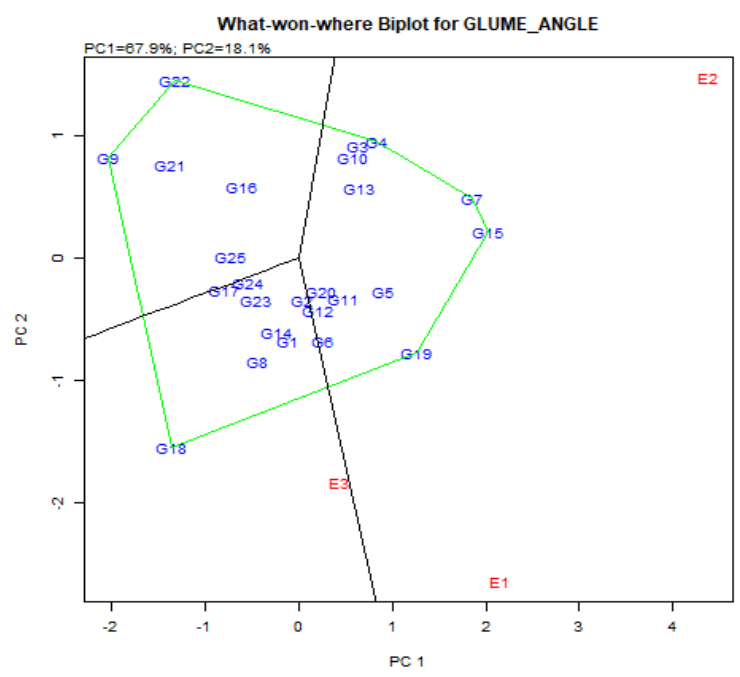

c) Angle of glume opening

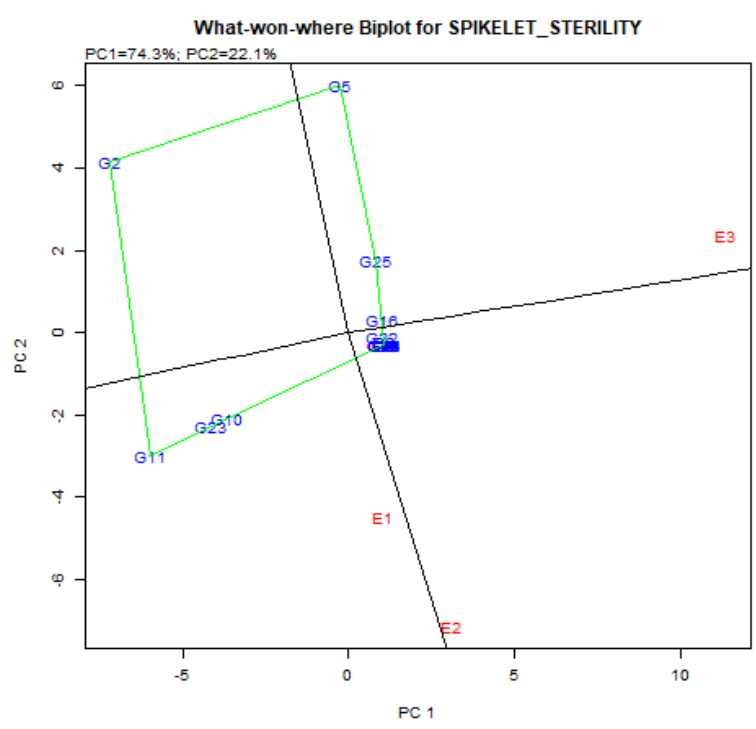

b) Spikelet sterility

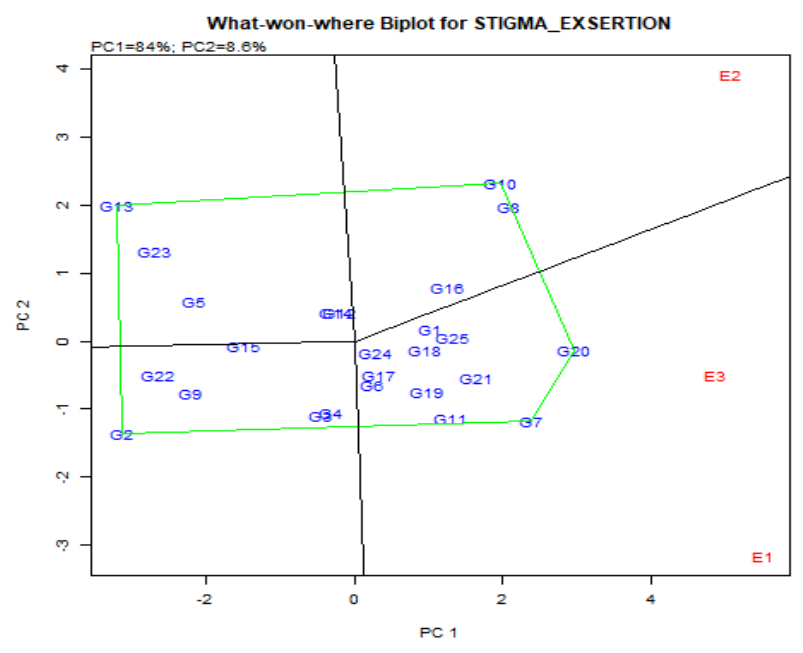

d) Stigma exsertion 


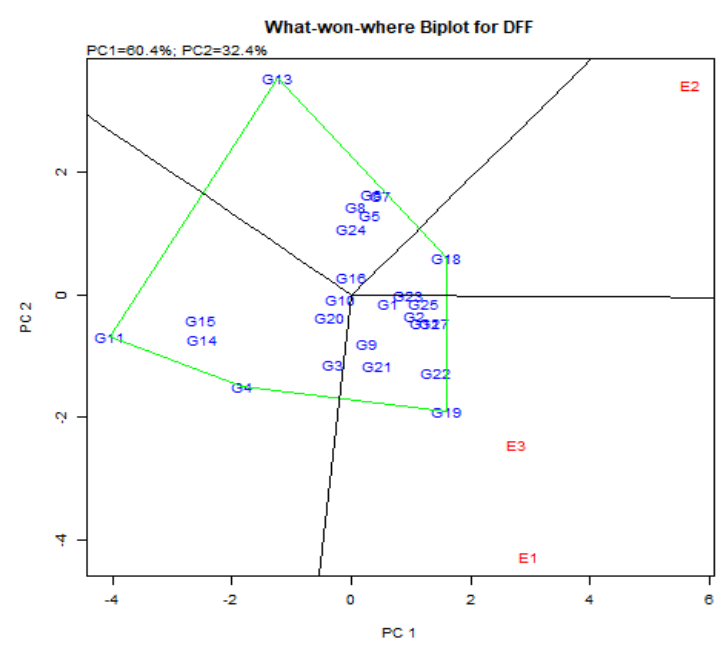

e) Days to $50 \%$ flowering

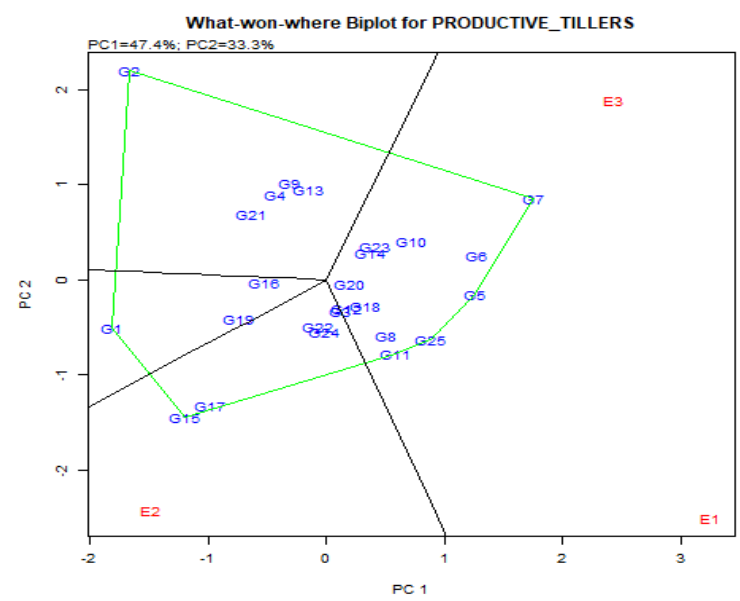

g)Plant height

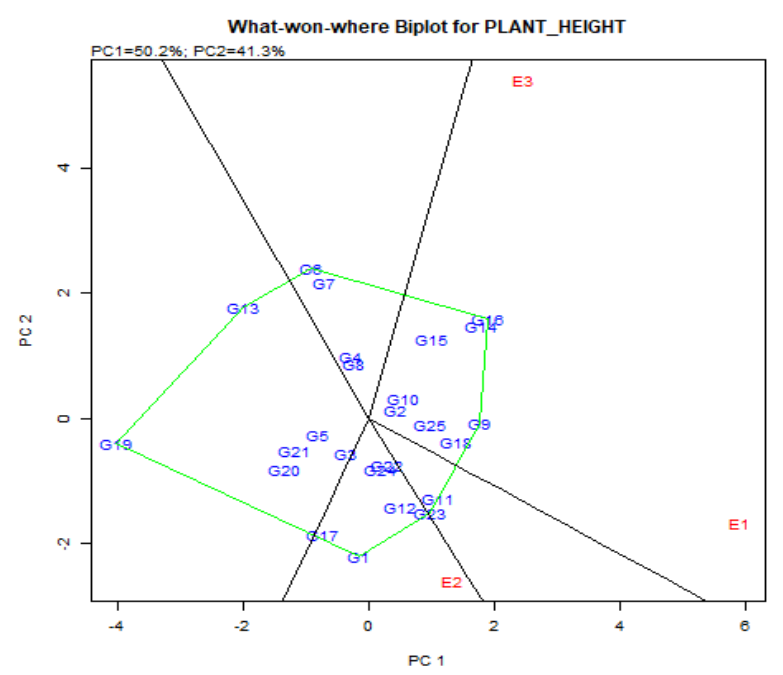

f) Panicle length

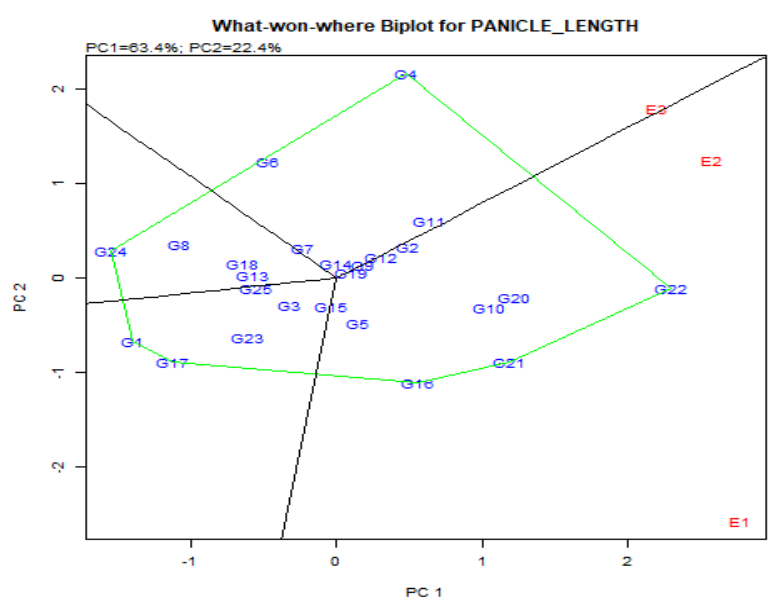

h)Panicle length 


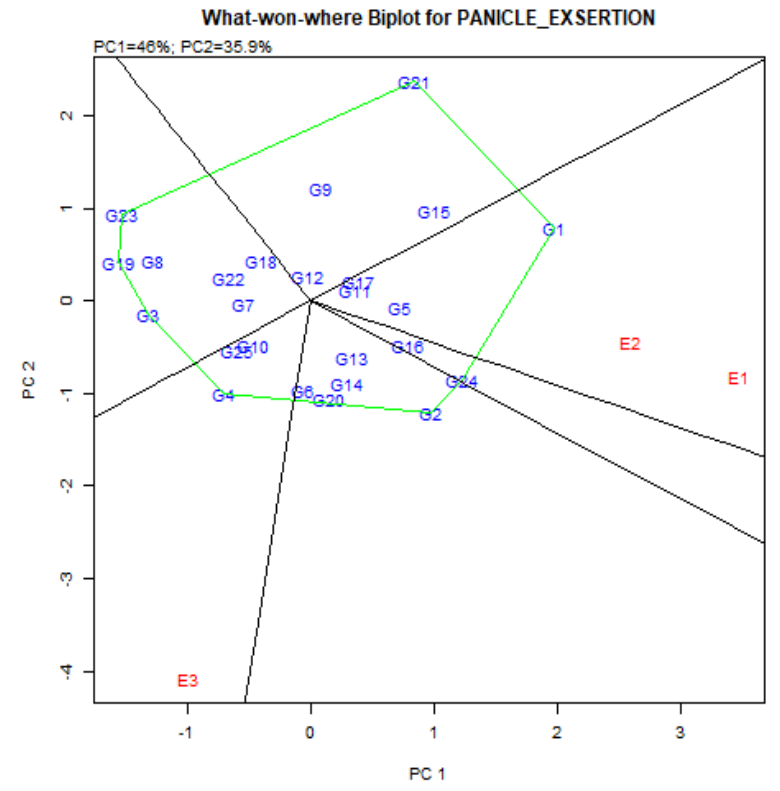

\section{i) Panicle exsertion}

Fig.3 Comparison of environments with ideal environment

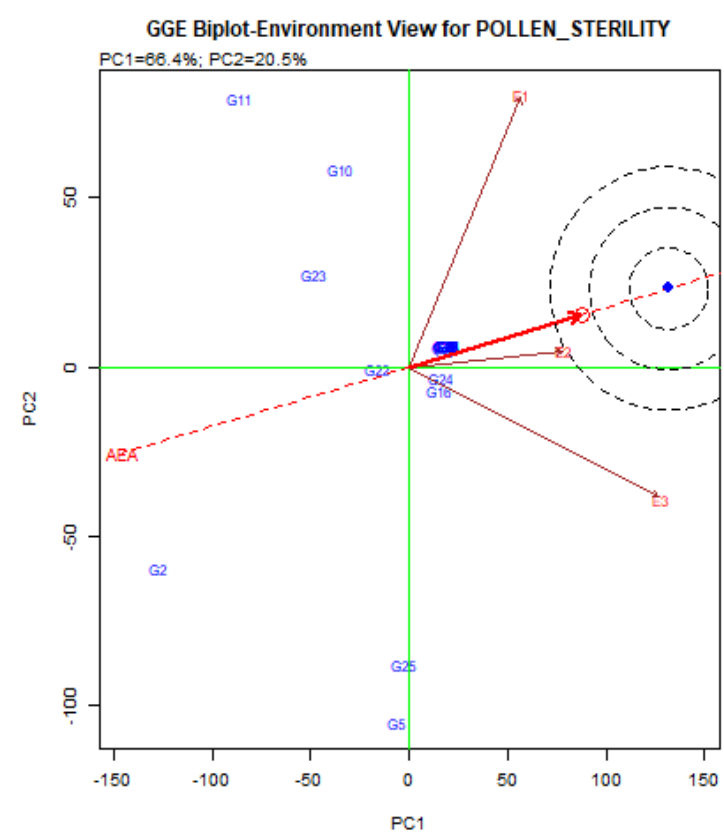

a) Pollen sterility

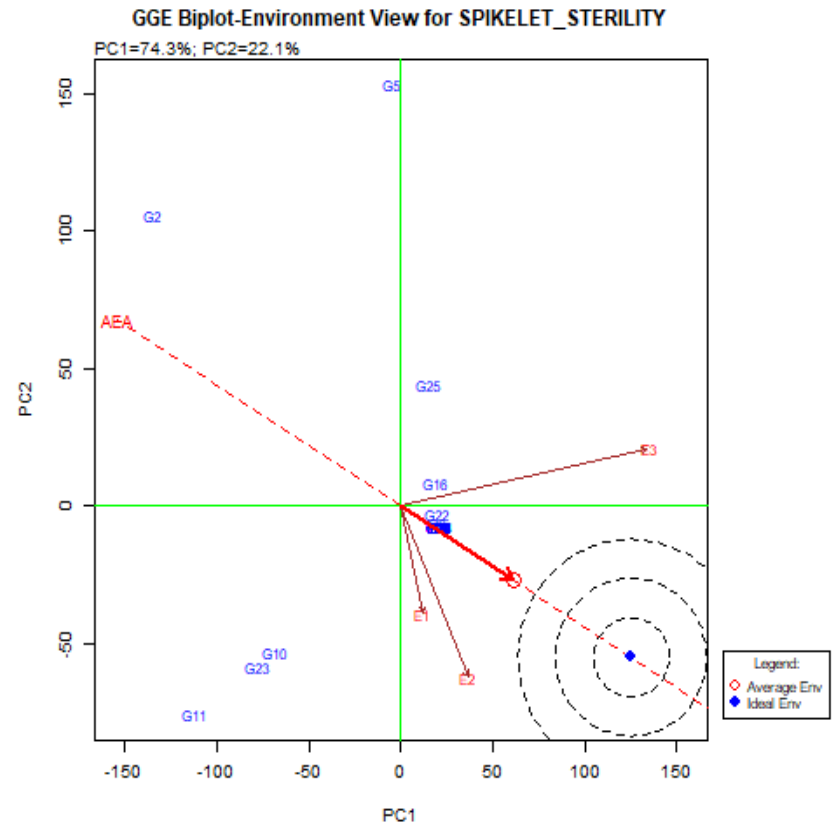

b) Spikelet sterility 
Fig.4 GGE biplot showing relationship among the test environments

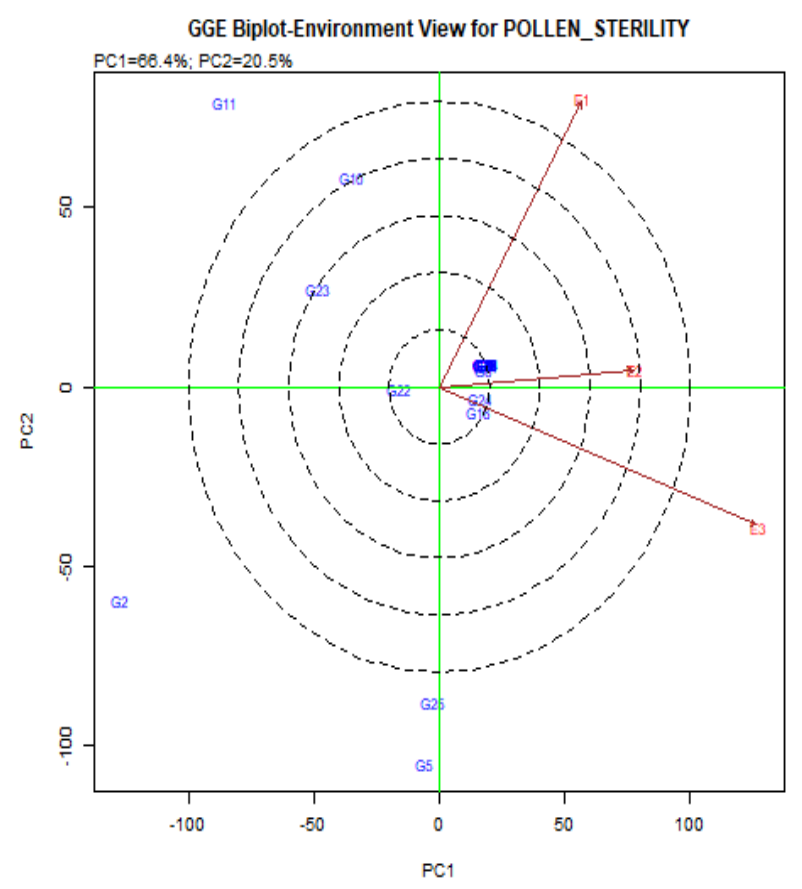

a) Pollen sterility

Balestre et al., (2010) evaluated 20 genotypes of upland rice and identified two cultivars viz., BRS Pepita and MG1097 as ideal in terms of stability and adaptability. Donoso-Ñanculao et al., (2015) using GGE biplot technique identified that Quila 241319 was the best genotype across three locations for grain yield and stability in temperate climate.

Jay Laxami et al., (2017) did 'which won where' analysis and found that mega environment 1 with two locations E2 and E3 had G10 as winning genotype, and in E1 and E4 falling in mega environment 2, G8 was the winning genotype. Farshadfar et al., (2013) identified genotype G17 (X96TH41K4) in environment E1, E3, E4 and E6, G12 (X96TH46) in environments E5, E7 and E8 and G19 (FLIP-82-115) for environment of E2 as the winning genotypes respectively in chickpea. In soybean, genotypes C 17 (PS 1556) and C 11 (VLS 89) were identified as winning genotypes for grain yield at Majhera,

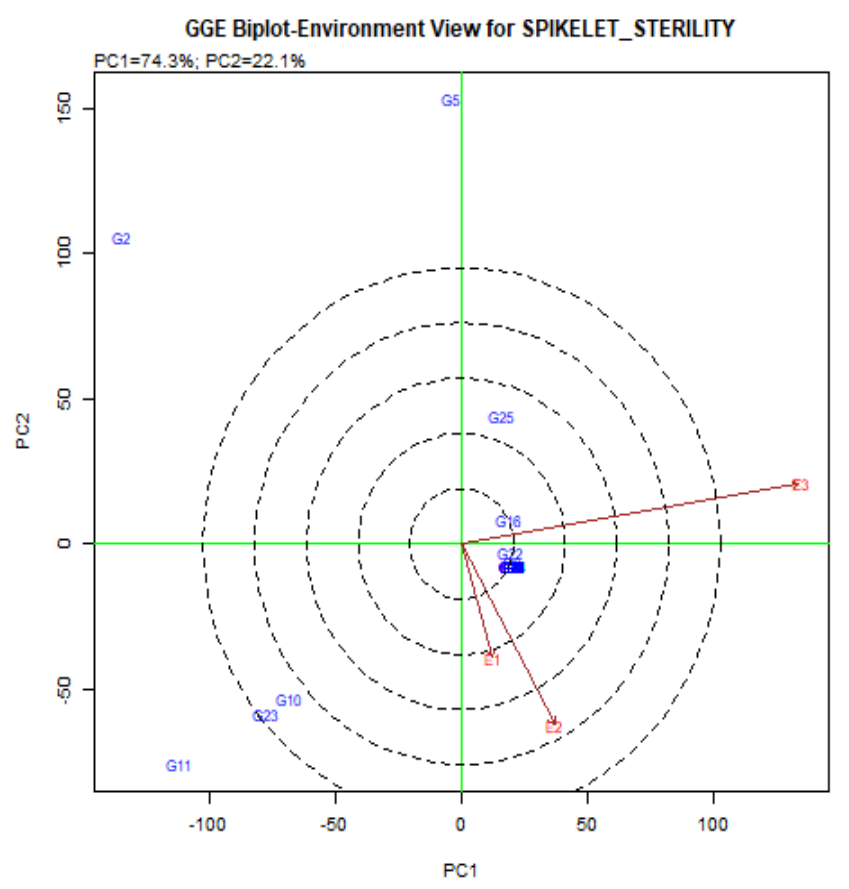

b) Spikelet sterility

Palampur and Almora and C 34 (VLS 59) at Bajaura by Bhartiya et al., (2017).

\section{Evaluation of environments based on GGE biplot}

In two line breeding of hybrid rice, particularly using Thermosensitive Genic Male Sterility system, identifying suitable locations for hybrid seed production is crucial where the sterility inducing temperature is prevailing during the critical stages. Hence the trait pollen and spikelet sterility \% were used as selection criterion for evaluation of environments in our study. Figure 3 depicts the representative and discriminative ability of the locations studied. The cosine of angle between environment vector and the Average Environment Axis (AEA) helps to identify the correlation between the genotype performance in that environment and across the environment (Yan et al., 2007). The length of the vector of the test environment measures 
the ability to discriminate genotypes in the test environment. Test environments making small angle with the AEA was considered as the most representative environment (Oyekunle et al., 2017).

Accordingly, the environment E2 (Aduthurai) was placed nearer to the ideal environment with long vector as well as small angle with the AEA followed by E1 (Coimbatore) and E3 (Ambasamudram) for pollen sterility and spikelet sterility. Thus E2 (Aduthurai) was ideal environment for both pollen and spikelet sterility was considered as stable and suitable environment for all genotypes. In earlier studies by Sairekha et al., (2018), the same location Aduthurai was identified to be stable for both pollen and spikelet sterility in TGMS lines.

The environment E3 (Barisal) was reported as 'ideal' environment followed by E1(Gazipur), E2 (Comilla) and E5 (Satkhira) for stability of yield in hybrid rice by Akter et al., (2015). Environment E2 (Late transplanting) and E3 (System of rice intensification) were reported as closest to the ideal environment for basmati rice by Jay Laxami et al., (2017). Susanto et al., (2015) reported that among the five environments tested, three environments viz., E1 (Subang, DS 2012), E2 (Karawang, DS 2012), and E3 (Indramaru, DS 2012) were identified to be good for discriminative and representative environment for yield trait of high Fe content rice lines.

\section{Relationship among environments}

The correlation between the environments represented by the vectors of all three environments facilitates the determination of the relationship between the environments. Cosine of angle between the locations shows the correlation among them (Yan, 2001). Acute angle represents positive, obtuse angle depicts negative and large $\mathrm{G} \times \mathrm{E}$ and right angle represents no correlation between environments (Yan and Tinker, 2006).

GGE biplot for relationship among the tested locations for pollen and spikelet sterility \% is shown in Figure 4. In case of pollen and spikelet sterility, acute angle was observed among the three environments, thus it shows there was strong positive correlation between the environments.

In the present study, TNAU $14 \mathrm{~S}$ (G1), TNAU 100S (G3), TNAU 101S (G4), TNAU 116S (G7), TNAU 124S (G8), TNAU 135S (G9), TNAU 18S (G12), TNAU 139S (G13), TNAU 143S (G15), TNAU 147S (G17), TNAU 151S (G18), TNAU 45S (G19), TNAU 46S (G20) and TNAU 67S (G21) were best performing and stable genotypes in terms of pollen sterility $\%$ and spikelet sterility \%. Among them, TNAU 151S (G18) and TNAU 124S (G8) are found to be stable and best performing in terms of productive tillers, TNAU 139S (G13) for panicle exsertion, TNAU 45S (G19) for angle of glume opening, TNAU 67S (G21) for stigma exsertion, TNAU 46S (G20) for stigma exsertion and panicle length, respectively. High and stable pollen and spikelet sterility could be achieved in Environment E2 (Aduthurai) and it was considered as stable environment. Thus the present investigation has led to identification of ideal TGMS lines and ideal environment for hybrid seed production using two-line breeding.

\section{References}

Akter, A., Hasan, M. J., Kulsum, U., Rahman, M. H., Khatun, M., \& Islam, M. R. (2015). GGE biplot analysis for yield stability in multi-environment trials of promising hybrid rice (Oryza sativa L.). Bangladesh Rice Journal, 19(1), 1-8.

Balestre, M., Santos, V. B. D., Soares, A. A., 
\& Reis, M. S. (2010). Stability and adaptability of upland rice genotypes. Crop Breeding and Applied Biotechnology, 10(4), 357-363.

Bhartiya, A., Aditya, J. P., Kumari, V., Kishore, N., Purwar, J. P., Agrawal, A., \& Kant, L. (2017). GGE biplot \& AMMI analysis of yield stability in multi-environment trial of soybean [Glycine max (1.) merrill] genotypes under rainfed condition of north western himalayan hills. JAPS, Journal of Animal and Plant Sciences, 27(1), 227-238.

Divya Balakrishnan, Subrahmanyam D., Badri J., Raju A.K., Rao Y.V.,, Beerelli K., Mesapogu S., Surapaneni M., Ponnuswamy R., Padmavathi G., Ravindra Babu V. and Neelamraju S. (2016). GenotypexEnvironment Interactions of Yield Traits in Backcross Introgression Lines Derived from Oryza sativa cv.Swarna/Oryza nivara. Frontiers in Plant Science, 7 :

Donoso-Ñanculao, G., Paredes, M., Becerra, V., Arrepol, C., \& Balzarini, M. (2016). GGE biplot analysis of multienvironment yield trials of rice produced in a temperate climate. Chilean journal of agricultural research, 76(2), 152-157.

Ebdon, JS., and Gauch, H.G. 2002. Additive main effect and multiplicative interaction analysis of national turfgrass performance trials II. Cultivar recommendations. Crop Sci. 42: 497506.

Farshadfar, E., Rashidi, M., Jowkar, M. M., \& Zali, H. (2013). GGE Biplot analysis of genotypex environment interaction in chickpea genotypes. Eur. J. Exp. Biol, 3, 417-423.

IIRI. Plant Breeding (PB) Tools, version 1.3 (2013) International Rice Research Institute. Manila, Philippines, http://bbi.irri.org.
Jay Laxami, Bupesh Kumar and Razdan A.K. (2017). GGE Biplot Analysis of Genotype x Environment Interaction in Basmati Rice (Oryza sativa L.) Int.J.Curr.Microbiol.App.Sci, $\quad$ 6(12): 3345-3350.

Jiang, T., Li R., Sun, C., and Wang, X. 2002. Utilization of diverse rice ecotypes in heterosis breeding. Breeding science 52:107-113.

Kang, M. S. (Ed.). (2002).GenotypeEnvironment interaction: progress and prospects in Quantitative genetics, genomics, and plant breeding. $\mathrm{CAB}$ International, Willingford. 221-243

Kang, M. S., Prabhakaran, V. T., \& Mehra, R. B. (2004). Genotype-by-environment interaction in crop improvement. In Plant Breeding (pp. 535-572). Springer, Dordrecht.

Khalil, I.A., Rahman, H.U., Rehman, N.U., Arif, M., Khalil, H.I., Iqbal, M., Hidayatullah, Afridi, K., Sajjad, M., and Ishaq, M. 2011. Evaluation of maize hybrids for grain yield Stability in north-west of Pakistan. Sarhad $j$. Agric. 27(2): 213-2181

Khodadad, M., Imeni, S. H., \& Zare, M. (2011). Stability Analysis of Rice Genotypes Based GGE biplot Method in North of Iran. Journal of Applied Sciences Research, 7(11), 1690-1694.

Mitrovic, B., Stanisavljevi, D., Treski, S., Stojakovic, M., Ivanovic, M., Bekavac, G., and Rajkovic, M.2012. Evaluation of experimental maize hybrids tested in multi-location trials using AMMI and GGE biplot analyses. Turkish Journal of Field Crops, 17(1): 35-40.

Nassir, A. L., \& Ariyo, O. J. (2011). Genotype $\mathrm{x}$ environment interaction and yieldstability analyses of rice grown in tropical inland swamp. Notulae Botanicae Horti Agrobotanici ClujNapoca, 39(1), 220-225.

Sairekha, K., Arumugachamy, S., Suresh, R., 
Saraswathi, R. and Kumar, M. 2018. GGE Biplot Analysis for Thermo Sensitive Genic Male Sterile Lines of Rice (Oryza sativa L.) in MultiEnvironment Trials. International Journal of Current Microbiology and Applied Sciences 7(1)-186-195.

Singh, V.K., Upadhyay, P., Sinha, P., Mall, A.K., Aiswal, S.K., Singh, A., Ellur, K.R., Biradar, S., Sundaram, R.M., Singh, S., Ahmed, I., Mishra, B., Singh, A.K. and Kole, C.2011. Determination of genetic relationships among elite thermosensitive genic male sterile lines (TGMS) of rice (Oryza sativa L.) employing morphological and simple sequence repeat (SSR) markers. Journal of Genetics 90(1):107-113.

Standard Evaluation System, IRRI, 2001.

Susanto, U., Rohaeni, W.R., Sarah, B., Johnson, S.B. and Jamil A. 2015. GGE biplot analysis for genotype $\mathrm{x}$ environment interaction on yield trait of high $\mathrm{Fe}$ content rice genotypes in Indonesian irrigated environments.
AGRIVITA 37 (3):265-275.

Yan, W. 2001. GGE biplot - A windows application for graphical analysis of multi-environment trial data and other types of two way data. Agron. J. 93: 1111-1118.

Yan, W. and Hunt L.A. 2002. GGE biplot analysis of muti- environment trial data, In M.S. Kang, ed. Quantitative genetics, Genomics and Plnat breeding. $\mathrm{CAB}$ International, Willingford.

Yan, W. and Kang, M.S. 2003. GGE biplot analysis: A graphical tool for breeders, geneticists, and agronomists.CRC Press, Boca Raton, FL.

Yan, W., \& Tinker, N. A. (2006). Biplot analysis of multi-environment trial data: Principles and applications. Canadian journal of plant science, 86(3), 623-645.

Yan, W., Hunt, L. A., Sheng Q. and Szlavnics Z. 2000. Cultivar evaluation and megaenvironment investigation based on GGE biplot. Crop Sci. 40:597-605.

\section{How to cite this article:}

Vinodhini, M., R. Saraswathi, P. L. Viswanathan, S. Arumugachamy, D. Sassikumar and Suresh, R. 2019. GGE Biplot Analysis in Thermo Sensitive Genic Male Sterile Lines of Rice (Oryza sativa L.) across Multiple Environments. Int.J.Curr.Microbiol.App.Sci. 8(10): 24922509. doi: https://doi.org/10.20546/ijcmas.2019.810.290 